\title{
Search for small trans-Neptunian objects by the TAOS project
}

\author{
W.P. Chen ${ }^{1}$, C. Alcock ${ }^{2}$, T. Axelrod ${ }^{3}$, F.B. Bianco ${ }^{2,6}$, Y.I. Byun ${ }^{4}$, \\ Y.H. Chang ${ }^{1}$, K.H. Cook $^{5}$, R. Dave ${ }^{6}$, J. Giammarco ${ }^{6}$, D.W. Kim ${ }^{4}$, \\ S.K. King ${ }^{7}$, T. Lee $^{7}$, M. Lehner ${ }^{2}$, C.C. Lin ${ }^{1}$, H.C. Lin ${ }^{1}$, J.J. Lissauer ${ }^{8}$, \\ S. Marshall ${ }^{9}$, N. Meinshausen ${ }^{10}$, S. Mondal ${ }^{1}$, I. de Pater ${ }^{10}$, \\ R. Porrata ${ }^{10}$, J. Rice ${ }^{10}$, M.E. Schwamb ${ }^{6,11}$, A. Wang ${ }^{7}$, S.Y. Wang ${ }^{7}$, \\ C.Y. Wen ${ }^{7}$ and Z.W. Zhang ${ }^{1}$ \\ ${ }^{1}$ Institute of Astronomy, National Central University, Taiwan, \\ email: wchen@astro.ncu.edu.tw \\ ${ }^{2}$ Harvard-Smithsonian Center for Astrophysics, USA \\ ${ }^{3}$ University of Arizona, USA \\ ${ }^{4}$ Department of Astronomy, Yonsei University, Korea \\ ${ }^{5}$ IGPP, Lawrence Livermore National Laboratory, USA \\ ${ }^{6}$ Department of Physics \& Astronomy, University of Pennsylvania, USA \\ ${ }^{7}$ Institute of Astronomy and Astrophysics, Academia Sinica, Taiwan \\ ${ }^{8}$ NASA Ames Research Center, USA \\ ${ }^{9}$ Stanford Linear Accelerator Center, USA \\ ${ }^{10}$ University of California, Berkeley, USA \\ ${ }^{11}$ California Institute of Technology, Pasadena, CA
}

\begin{abstract}
The Taiwan-America Occultation Survey (TAOS) aims to determine the number of small icy bodies in the outer reach of the Solar System by means of stellar occultation. An array of 4 robotic small $(\mathrm{D}=0.5 \mathrm{~m})$, wide-field (f/1.9) telescopes have been installed at Lulin Observatory in Taiwan to simultaneously monitor some thousand of stars for such rare occultation events. Because a typical occultation event by a TNO a few km across will last for only a fraction of a second, fast photometry is necessary. A special CCD readout scheme has been devised to allow for stellar photometry taken a few times per second. Effective analysis pipelines have been developed to process stellar light curves and to correlate any possible flux changes among all telescopes. A few billion photometric measurements have been collected since the routine survey began in early 2005. Our preliminary result of a very low detection rate suggests a deficit of small TNOs down to a few $\mathrm{km}$ size, consistent with the extrapolation of some recent studies of larger $(30-100 \mathrm{~km})$ TNOs.
\end{abstract}

Keywords. Photometry; occultations; Trans Neptunian objects; comets, discovery; surveys

\section{The Taiwan-America Occultation Survey (TAOS) Project}

As of September 2006, there are over 1000 Trans-Neptunian Objects (TNOs) known (www.boulder.swri.edu/ekonews). Because of their large distances and small sizes, only the brightest, hence the largest, TNOs could be detected by their reflected sunlight. The faintest objects detected so far correspond to a size of about a few tens of $\mathrm{km}$. There seems a deficit of TNOs in this size range in comparison with larger ones, perhaps as a result of collisional disruption (Bernstein et al. 2004). To determine the number of even smaller TNOs, TAOS has implemented an array of 4 small (aperture $0.5 \mathrm{~m}$ ), wide-field (f/1.9) telescopes at Lulin Observatory in Taiwan to monitor for chance stellar occultation by 


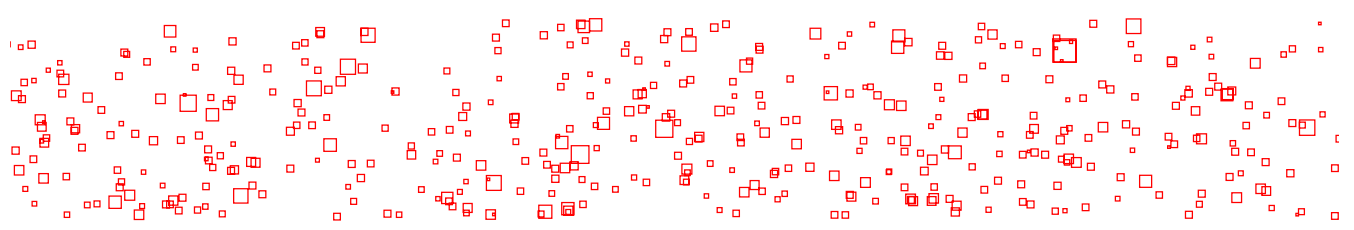

Figure 1. The photometry aperture mask to process the TAOS data. Each star has a different aperture size for a maximum signal-to-noise light curve. The position of the aperture changes according to the centroid of the star to accommodate image motion.

TNOs (King et al. 2001; Alcock et al. 2003). TNOs as small as 1-2 km across should be detectable by TAOS. The occurrence rate of occultation events will provide constraints on the number and spatial distribution of TNOs.

Occultation by a TNO a few $\mathrm{km}$ across lasts for a fraction of a second, so rapid photometry is necessary. The 4 TAOS telescopes are located with a maximum separation of $\sim 100 \mathrm{~m}$, too close to detect the shadow speed by timing difference at different telescopes, but allowing coincidence detection by multiple telescopes to reduce the false alarm rate. In addition to the TNO science, the huge TAOS database, some 100 GB per night, should be valuable in other studies such as stellar variability. Our robotic system is also responding to gamma-ray bursts.

\section{Current Status of the TAOS Project}

\subsection{Data Acquisition and Adaptive Aperture Photometry}

The TAOS CCD camera, instead of reading out the entire chip as in a regular imaging observation, continuously integrates (pause) and reads out a block of pixels (shift) one at a time, while the shutter remains open and the telescope tracks on the target field. This electronic "pause-and-shift" scheme in effect produces a sequence of snapshots and allows for stellar photometry at a rate up to a few hertz (Chen et al. 2003). The image thus obtained is crowded because all the stars in the field of view are "folded" into each readout block. The shift of electrons and the open shutter also cause a star to leave a streak, which interferes with the signal of a "nearby" star that appears to be adjacent in the readout block, though the two stars in fact may be widely separated in the sky. In addition, photons from either a neighboring star or a patch of sky are recorded at different times.

A photometry pipeline has been developed to deal with the crowdedness and temporal/spatial blending in the data. For each star, an optimal aperture size is used to minimize contamination from neighboring stars or streaks. An appropriate patch of sky is chosen for subtraction. An "aperture mask" is then applied to measure the fluxes of all the stars (Fig. 1). The position of the aperture keeps centered on, i.e., being adaptive to, the peak pixel of a star from one readout block to another, so as to compensate for image motion. This Adaptive Aperture Photometry routine uses square apertures so accuracy is compromised - not a serious problem because TAOS images with a $3^{\prime \prime}$ pixel scale are undersampled - but it is very efficient, capable in real time to process about a thousand stars sampled at $5 \mathrm{~Hz}$. Other analysis methods are being evaluated.

\subsection{Event Detection - The Rank Statistics}

Figure 2 shows the occultation of the star TYC 076200961 by the asteroid (286) Iclea observed on 6 February 2006. TAOS observed this predicted event successfully with 3 telescopes. Iclea is known to have a diameter of $97 \mathrm{~km}$, and the TAOS system detected the event readily. 


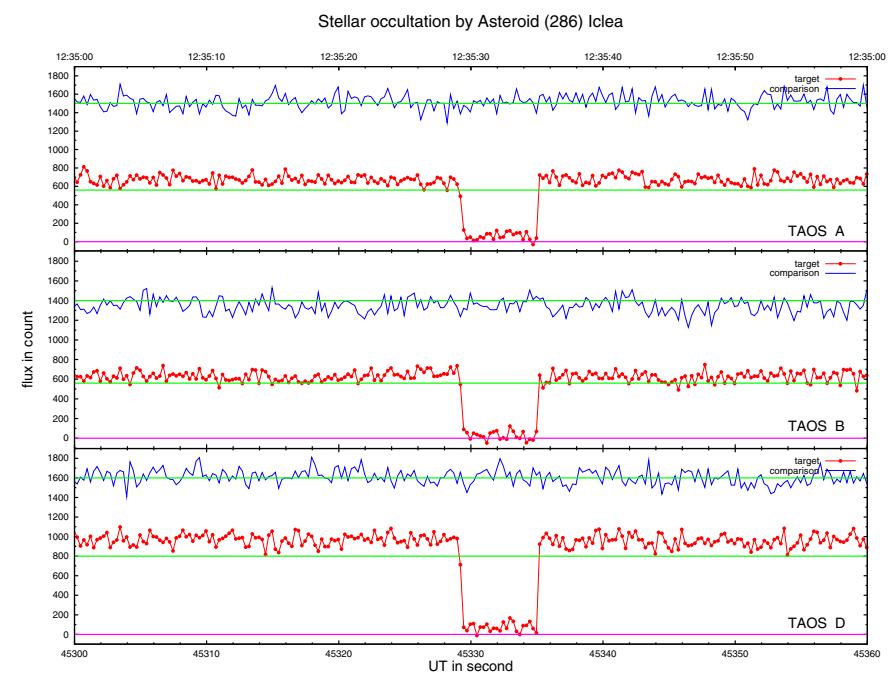

Figure 2. A showcase data of the star TYC $076200961\left(\mathrm{~m}_{\mathrm{V}} \sim 11.83 \mathrm{mag}\right)$ occulted by (286) Iclea $\left(\mathrm{m}_{\mathrm{V}} \sim 14.0 \mathrm{mag}\right.$ at the time) on 6 February 2006 observed by 3 TAOS telescopes. Data were taken at $4 \mathrm{~Hz}$, and the duration of the event was estimated to be $\approx 5.75 \mathrm{~s}$.

For short events, e.g., within one data point, we employ a nonparametric approach to identify simultaneous flux drops in stellar light curves. The flux at a given time is ranked among all data points. The rank statistic is then the product of the ranking orders of all telescopes. For example, if there are $N$ data points from one telescope, the lowest flux has the rank $R=1$ and the highest $R=N$. The rank statistic for data point $i$ is then

$$
Z_{i}=-\log \left(\prod_{k=1}^{N_{\text {tel }}} \frac{R_{i}^{k}}{N}\right)=\sum_{k=1}^{N_{\text {tel }}}\left(-\log \frac{R_{i}^{k}}{N}\right),
$$

where $N_{\text {tel }}$ is the total number of telescopes, and both the multiplication $\prod$ and summation $\sum$ are over $N_{\text {tel }}$. The quantity $Z$ approximates to a Gamma distribution, if the noise between telescopes is independent. A probable occultation event would stand out as an "outlier" against an otherwise random distribution.

\subsection{Expected Event Rate}

The detected rate of TNO occultation events depends on the actual occurrence rate and the detectability. Relevant parameters include: (1) The surface number density (our goal) and angular size distribution of TNOs. (2) The surface number density and angular size distribution of background stars. Both these depend on the Galactic line of sight. With our current instrument setup, the $5 \mathrm{~Hz}$ observations reach about $\mathrm{R}=14 \mathrm{mag}$, which gives within the $3 \mathrm{deg}^{2}$ field of view typically several hundred to a few thousand stars in a target field. A dense field would be favorable for occultation but would create images too crowded for analysis. The angular size of a star can be estimated, for example, by its optical and infrared colors (van Belle 1999). Most stars have an angular size less than 0.1 milliarcsecond (mas). For reference, a TNO at $50 \mathrm{AU}$ with an angular size of 0.1 mas has a physical diameter of $\sim 4 \mathrm{~km}$. Items (1) and (2) together specify the probability of area overlap (geometric consideration for occultation), plus the diffraction effect (King et al. 2006; Lehner et al. 2006), which tends to smooth out the flux drop and becomes important for small or distant TNOs. The extrapolated number density of small $(>1 \mathrm{~km})$ TNOs varies widely, ranging from $10^{2}$ to $10^{6}$ per $\operatorname{deg}^{2}$ (Bernstein et al. 2004). This amounts to 
about 1 event per few days to 1 event over a few years. (3) The shadow speed. Observing toward the opposition for example, as opposed to the quadrature, maximizes the chance of occultation but the event duration is short so difficult to detect. (4) CCD integration time and sampling rate, etc. These affect the limiting magnitude, hence the number of stars observable, and the capability to resolve a short flux drop.

Since 2005, TAOS with 3 telescopes has collected some billions of photometric measurements. Preliminary analysis shows a very low detection rate. A suspected event is shown in Fig. 3 where a relatively faint star $\left(\mathrm{R} \sim 13.5 \mathrm{mag}, \mathrm{RA}=13^{\mathrm{h}} 46^{\mathrm{m}} 26.7^{\mathrm{s}}\right.$ and Decl $=-10^{\circ} 50^{\prime} 31^{\prime \prime}$ ) was detected to have a $39 \%, 56 \%$, and $50 \%$ flux drop, respectively, in 3 telescopes. The probability is, according to the Gamma approximation, $1.7 \times 10^{-7}$ that the coincident flux drops were caused by chance. Given our high data rate, however, we cannot be highly confident that this was an actual occultation event. A fourth telescope, which is expected to be in service in early 2007, would increase greatly the credibility of a detection. Our result suggests a deficit down to km-sized TNOs. It is not clear at the moment how our low detection rate reconciles with the claim of numerous even smaller $(<100 \mathrm{~m})$ TNOs (Chang et al. 2006).
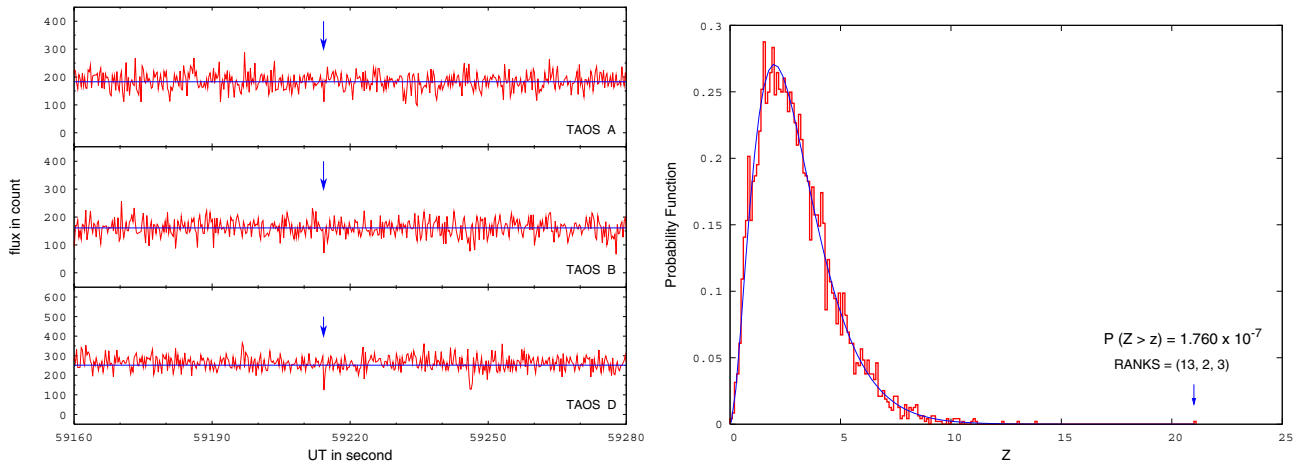

Figure 3. (Left) Light curves of a suspected TNO occultation event observed on 7 April 2005. (Right) Histogram of the rank statistics (2.1). This event ranks $(13,2,3)$, respectively, in 3 telescopes out of 4728 data points sampled every $0.25 \mathrm{~s}$. The smooth line is the Gamma distribution. The arrow marks the outlier event.

The NCU group acknowledges the NSC grant 95-2119-M-008-028. KHC's work was performed under the auspices of the US DOE, by the University of California, LLNL under contract No. W-7405-Eng-48.

\section{References}

Alcock, C., et al. 2003, Earth, Moon $\mathcal{E}$ Planets 92, 459

Bernstein, G.M., et al. 2004, ApJ 128, 1364

Chang, H.K., et al. 2006, Nature 442, 660

Chen, W.P., et al. 2003, Baltic Ast. 12, 568

King, S.K. 2001, in Small-Telescope Astronomy on Global Scales, eds. W.P. Chen, C. Lemme, \& B. Paczynski, ASP Conf. Ser, 246, 253

King, S.K., et al. 2006, in: W. Ip \& A. Bhardwaj (eds.), Adv. in Geosci., Vol. 3 (World Sci.) p. 345

Lehner, M.J., et al. 2006, Astron. Nach 327, 814

Liang, C.L., et al. 2004, Stat. Sci. 19, 265

van Belle, G.T. 1999, PASP 111, 1515 